\title{
Insights into oxygen fugacity and charge effects on diffusion from thermal diffusion experiments
}

\author{
X. B. LIN $^{1}$, C. C. LUNDSTROM ${ }^{1}$, K. BRÜCKEL ${ }^{1}$, M. J. \\ KRAWCZYNSKI ${ }^{2}$, C. E. LESHER ${ }^{3}$ \\ ${ }^{1}$ University of Illinois at Urbana-Champaign, Urbana, USA \\ (xlin29@illinois.edu; lundstro@illinois.edu; \\ bruckel2@illinois.edu) \\ ${ }^{2}$ Washington University in St. Louis, St. Louis, USA \\ (mikekraw@wustl.edu) \\ ${ }^{3}$ University of California, Davis, USA \\ (celesher@ucdavis.edu) \\ Applications of thermal diffusion, mass diffusion driven \\ by a temperature gradient, range from basic understanding of \\ materials such as melt sturcture to possible roles in element \\ and isotopic fractionations during rock forming processes. \\ Here we use new temperture gradient piston cylinder (PC) \\ experiments ( $\mathrm{T}$ gradients determined by the spinel growth \\ method) along with previous experiments ${ }^{[1]}$ to not only \\ examine elemental and isotopic fractionation but also \\ characterize valence state changes of redox active elements \\ like Fe and V. \\ We performed thermal diffusion experiments from $1 \mathrm{hr}$ to \\ $100 \mathrm{hr}$ in $\mathrm{PC}$ at $1.5 \mathrm{GPa}$ and hot spot temperature from 1650 \\ ${ }^{\circ} \mathrm{C}$ to $1560{ }^{\circ} \mathrm{C}$ with about $200{ }^{\circ} \mathrm{C}$ drop over $\sim 4 \mathrm{~mm}$ capusle. \\ For these, we used a basalt doped with either $\sim 1 \mathrm{wt} . \% \mathrm{Cr}$ or $\mathrm{V}$ \\ oxides.XANES spectroscopy at APS determined the valence \\ state of $\mathrm{V}$ and Fe along the length of the capsules. The \\ valence states of $\mathrm{Fe}$ and $\mathrm{V}$ are predominantly +2 and +3 for \\ basaltic thermal diffusion experiments near the CCO buffer \\ along the temperature gradient ( $\mathrm{Pt}+$ graphite configuration). \\ Notably, thermal diffusion in rhyolite liquid shows the \\ existence of $\mathrm{Fe}^{3+}$ which is most likely due to the composition \\ effect on Fe reduction potential. This provides a constraint for \\ redox reaction thermodynamic datasets in the silicate melts at \\ high temperature ${ }^{[2,3]}$. \\ We also used $193 \mathrm{~nm}$ laser ablation ICP-MS to determine \\ major and trace element abundances in these experiments for \\ rhyolitic to basaltic composition. Soret coefficients, which \\ describes the overall separation by temperature gradient, of \\ rare earth elements produce a negative Eu anomaly relative to \\ its neighboring elements in the basaltic melts. This is \\ attributed to the presence of $\mathrm{Eu}^{2+}$, and thus Soret behavior \\ between $2+$ cation and other $\mathrm{REE}^{3+}$. The valence state \\ dependence of Soret coefficient of trace elements in basaltic \\ melts is observed and can be potentially applied to the \\ determination of the valence state of multi-valent elements. \\ [1] Lesher and Walker 1986, 1991. [2] Kavner et al., 2007. \\ [3] Toplis et al., 2002.
}

